Check for updates

Cite this: Phys. Chem. Chem. Phys., $2022,24,624$

Received 5th November 2021,

Accepted 10th December 2021

DOI: $10.1039 / \mathrm{d} 1 \mathrm{cp} 05061 \mathrm{c}$

rsc.li/pccp

\section{Integration of global ring currents using the Ampère-Maxwell law $\dagger$}

\author{
Raphael J. F. Berger, (D)*a Maria Dimitrova, (D) ${ }^{\text {ab }}$ Rinat T. Nasibullin, ${ }^{c}$ \\ Rashid R. Valiev ${ }^{\mathrm{bc}}$ and Dage Sundholm (D)*b
}

\begin{abstract}
Magnetically induced ring currents are calculated from the magnetic shielding tensor by employing the Ampère-Maxwell law. The feasibility of the method is demonstrated by integrating the $z z$ component of the shielding tensor along the symmetry axis of highly symmetric ringshaped aromatic, antiaromatic and nonaromatic molecules. The calculated ring-current strengths agree perfectly with the ones obtained by integrating the current-density flux passing through a plane cutting half the molecular ring. The method can be used in combination with all electronic structure codes capable of calculating nuclear magnetic resonance (NMR) shielding tensors in general points in space. We also show that nucleus independent chemical shifts (NICS) along the symmetry axis are related to the spatial derivative of the strength of the global ring-current along the $z$ axis.
\end{abstract}

An external magnetic field perpendicular to the molecular plane of a planar ring-shaped molecule may induce a ring current around the molecule. ${ }^{1-6}$ The strength of the ring current is often used for investigating the aromatic character of the molecule and its degree of aromaticity. ${ }^{7,8}$ The ring-current strength can be estimated from the isotropic magnetic shielding calculated in discrete points in the vicinity of the molecule. ${ }^{9-13}$ Methods based on the nucleus independent chemical shifts have been extended to two and three dimensions using the isotropic shielding constant or the $z z$ component of the shielding tensor. ${ }^{14-18}$ Ring-current strengths can be directly calculated by integrating the current-density flux passing through a surface $\mathbf{S}$ that cuts the molecular ring current. ${ }^{19-21}$

$$
I_{S}=\iint_{S} \mathbf{J} \cdot \mathrm{d} \mathbf{S}
$$

In eqn (1), $\mathbf{J}$ is the current density, the dot represents its scalar product with the normal of the infinitesimal cross-section area, $\mathrm{d} \mathbf{S}$,

\footnotetext{
${ }^{a}$ Chemistry of Materials, Paris-Lodron University of Salzburg, Jakob-Haringerstr. 2A, A-5020 Salzburg, Austria. E-mail: raphael.berger@plus.ac.at

${ }^{b}$ Department of Chemistry, Faculty of Science, P.O. Box 55, A. I. Virtasen aukio 1, FI-00014 University of Helsinki, Finland. E-mail: sundholm@chem.helsinki.fi

${ }^{c}$ Tomsk State University, 36 Lenin Avenue, Tomsk 634050, Russia

$\dagger$ Electronic supplementary information (ESI) available: The Cartesian coordinates of the optimized molecular structures are reported. See DOI: 10.1039/d1cp05061c
}

and $I_{\mathrm{S}}$ is the strength of the current passing through the crosssection surface. To compute the strength of the global ring current, the two-dimensional integration domain has to begin in the center of the ring-current vortex and it ends at a very long distance from the molecule in the other spatial directions. This approach has been used for calculating ring-current strengths and ring-current pathways for a large number of molecules and it was reviewed recently. ${ }^{22-24}$

Here, we propose an alternative method to determine ringcurrent strengths. The presented approach is based on the integral form of the Ampère-Maxwell law that relates the integrated magnetic field around the boundary of a surface to the strength of the current passing through it. The integral form of the Ampère-Maxwell law reads

$$
\iint_{S} \mathbf{J} \cdot \mathrm{d} \mathbf{S}=\frac{1}{\mu_{0}} \oint_{\ell} \mathbf{B}_{\text {induced }} \cdot \mathrm{d} \ell,
$$

where $\mathbf{B}_{\text {induced }}$ is the magnetic field along the closed curve $\ell$ enclosing $\mathbf{S}$ and $\mu_{0}=1.25663706212(19) \times 10^{-6} \mathrm{~N} \mathrm{~A}^{-2}$ is the vacuum permeability. We investigate planar molecules such as benzene that lie in the $x, y$ plane and have an axial symmetry with the $z$ axis coinciding with the main symmetry axis. The chosen molecules belong to point groups having a main symmetry axis of the order of $\geq 3$ or to the $D_{2}$ point group, since this class of molecules has a vertical ring-current vortex for symmetry reasons and the central vortex stagnation line coincides in such molecules exactly with the $z$ axis. The external magnetic field is perpendicular to the molecular ring.

According to eqn (2) the strength of the ring current can be obtained by numerically integrating the $\sigma_{z z}$ component of the magnetic shielding tensor along the $z$ axis. Since the induced magnetic field vanishes at a very long distance from the molecule, the integration path can be closed by bending it far away from the molecule where it approaches the $z$ axis from the other side of the molecule. The induced magnetic field is obtained from the position dependent chemical shielding tensor $\boldsymbol{\sigma}$ by contracting with the external magnetic field $\mathbf{B}_{\text {external }}$ that is set parallel to the $z$ axis in this work. 


$$
\mathbf{B}_{\text {induced }}=-\boldsymbol{\sigma} \mathbf{B}_{\text {external }}
$$

The shielding tensor is computed in a number of discrete point along the $z$ axis and integrated numerically. For the studied molecules, the non-parallel components of the induced magnetic field vanishes. Thus, the method involves numerical integration of only the $z z$ component of the aromatic ringcurrent shielding (ARCS) function, whose shape has previously been used for estimating ring-current strengths. ${ }^{10,11,25-27}$ The ARCS idea has later been adopted, extended and generalized..$^{28-30}$ For molecules whose $x$ and $y$ components of the induced magnetic field do not vanish along the $z$ axis, the present approach leads to a small numerical deviation from the exact ring-current strength because the path integration does not follow the stagnation line of the central vortex. The currentdensity integration scheme employing a rectangular integration domain suffers from similar problems, because the boundary of integration domain does not always coincide with the center of the current-density vortex implying that whole ring current is not obtained or some of the returning ring current is considered. ${ }^{31}$ The deviations are usually small and can in both approaches be accounted for by using a slightly extended scheme.

The method is demonstrated by calculating the strength of the ring current of cyclobutadiene, benzene, cyclohexadiene, borazine, $p$-benzoquinone, hexadehydro ${ }^{12}$ annulene, porphyrin and isophlorin. The profile of the ring current of benzene is compared to the one obtained by integrating the current density.

The molecular structures were optimized at the density functional theory (DFT) level using the B3LYP functional, ${ }^{32-34}$ the def2-TZVP basis set, ${ }^{35}$ and the semiempirial D3-BJ dispersion correction. ${ }^{36}$ DFT optimizations of the ground-state molecular structures were performed with Turbomole. ${ }^{37}$ The Cartesian coordinates of the optimized molecular structures are given in the ESI. $\dagger$

Nuclear magnetic shielding tensors were calculated with the mpshift module of Turbomole. ${ }^{38}$ Magnetically induced current densities were calculated with the GIMIC program using basisset data and density matrices obtained in the NMR shielding calculations. ${ }^{19,21,39}$ Reference values for the ring-current strengths were obtained with GIMIC by numerically integrating the current-density flux passing the rectangular cross-section area through half the ring.

The ring-current strengths were also obtained from AmpèreMaxwell's circuital law by numerically integrating the $z z$ component of the nuclear magnetic shielding tensor along the $z$ axis. We use the five-point Boole's rule $\frac{2 h}{45}\left(7 f_{0}+32 f_{1}+12 f_{2}+32 f_{3}+7 f_{4}\right)$ to approximate the integral in each five-point element. ${ }^{40,41}$ We used an equidistant step length $h$ of 0.25 bohr between the grid points and $f_{i}$ are the $\sigma_{z z}$ value calculated in each grid point. We used 1001 equidistant integration points with the first one at $z=-100 \mathrm{bohr}$ and the last one at $z=100 \mathrm{bohr}$ from the molecular plane, because the induced magnetic field declines very slowly for large molecular rings sustaining a strong ring current.
Table 1 The ring-current strengths (in $n A T^{-1}$ ) obtained from AmpèreMaxwell's law, IAmpère (eqn (2)), and $I_{\text {flux }}$ (eqn (2)), which is obtained by integrating the current density passing though the cross section of half the molecular ring

\begin{tabular}{lrr}
\hline Molecule & $I_{\text {Ampère }}$ & \multicolumn{1}{c}{$I_{\text {flux }}$} \\
\hline Cyclobutadiene & -20.00 & -19.82 \\
Benzene & 11.94 & 12.04 \\
Cyclohexadiene & -0.65 & -0.47 \\
Borazine & 3.25 & 3.23 \\
$p$-Benzoquinone & -0.01 & 0.21 \\
Hexadehydro[12]annulene & -24.16 & -23.98 \\
Porphyrin & 27.59 & 27.03 \\
Isophlorin & -63.99 & -63.34 \\
\hline
\end{tabular}

The ring-current strengths obtained by integrating the current-density flux circling around the ring and the ones obtained using Ampère-Maxwell's law in Table 1 agree very well showing that the ring-current strength can be obtained with high accuracy from the magnetic shielding tensor. The deviation between the two values are less then $0.2 \mathrm{nA} \mathrm{T}^{-1}$ for most of the studied molecules. The deviations are 0.4-0.6 $\mathrm{nA} \mathrm{T}^{-1}$ for the porphyrinoids with very strong ring currents.

The ring-current strength of isophlorin is overestimated, because we use the B3LYP functional with only $20 \%$ Hartree-Fock (HF) exchange. A smaller paratropic ring current would be obtained when using a functional with at least $50 \%$ HF exchange or when using a range-separated functional. ${ }^{42,43}$ However, this does not affect the conclusions, since the two ring-current strengths for isophlorin agree well.

Application of Ampère-Maxwell's law to evaluate total induced ring current strengths also sheds light on the physical meaning of NICS values ${ }^{9,13}$ and their relation to the magnetically induced current density. For convenience, we define the integral over the path $\ell$ between the points $a$ and $b$ in the right-hand-side of eqn (2) as

$$
I_{\ell}[a ; b]=\frac{1}{\mu_{0}} \int_{a \in \ell}^{b \in \ell} \mathbf{B}_{\text {induced }} \cdot \mathrm{d} \ell,
$$

which can be generalized to any open or closed segment paths. For closed integration paths, the integral is independent of the integration boundaries $(a=b)$ implying that they can be omitted in the notation.

Evaluating the line integral in eqn (2) along a general rectangular path shown in Fig. 1 yields the strength of the magnetically induced current density passing through the surface enclosed by it. The contribution from the first part $\left(l_{1}\right)$ of the integration path starting at $\mathbf{r}_{0}=\left(0,0,-z_{0}\right)$ on the symmetry axis and ending at $\mathbf{r}_{1}=\left(0,0, z_{0}\right)$ is given by

$$
I_{l_{1}}\left[\mathbf{r}_{0} ; \mathbf{r}_{1}\right]=\frac{1}{\mu_{0}} \int_{-z_{0}}^{z_{0}} \sigma_{z z}(0,0, z) \mathrm{d} z \text {. }
$$

The contribution from the second part $\left(l_{2}\right)$ of the integration path that begins at $\mathbf{r}_{1}=\left(0,0, z_{0}\right)$ and ends at $\mathbf{r}_{2}=\left(\infty, 0, z_{0}\right)$ is obtained by

$$
I_{l_{2}}\left[\mathbf{r}_{1} ; \mathbf{r}_{2}\right]=\frac{1}{\mu_{0}} \int_{0}^{\infty} \sigma_{x z}\left(x, 0, z_{0}\right) \mathrm{d} x
$$




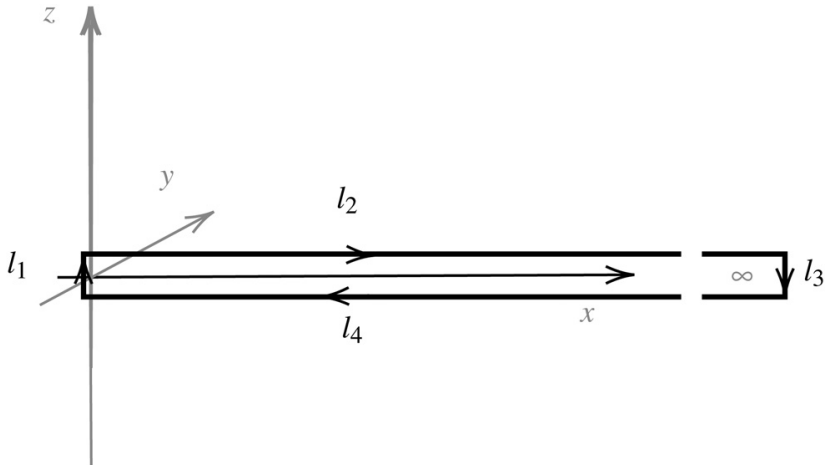

Fig. 1 An illustration of the integration path giving a relation between the magnetic shielding tensor $\left(\sigma_{z z}\right)$ along the path and the magnetically induced ring-current inside the closed loop ( $\left.l_{\text {inside }}\right)$.

where $\sigma_{x z}\left(x, 0, z_{0}\right)$ represents the induced magnetic shielding component in the $x$ direction along the integration line, when the external magnetic field is applied in the $z$ direction. The contribution from the third part $\left(l_{3}\right)$ of the integration path beginning at $\mathbf{r}_{2}=\left(\infty, 0, z_{0}\right)$ and ending at $\mathbf{r}_{3}=\left(\infty, 0,-z_{0}\right)$ vanishes because the path is infinitely far away from the molecule where the induced magnetic field dies out. The fourth integration interval $\left(l_{4}\right)$ is from $\mathbf{r}_{3}$ to $\mathbf{r}_{0}$. Since the linear integration paths of $l_{2}$ and $l_{4}$ are at the same distance from the molecular ring. They contribute equally due to the symmetry. Alternatively, the $l_{2}$ and $l_{4}$ contributions are equal, because the off-diagonal elements of the magnetic shielding tensor have different signs at $z$ and $-z$ and the integration interval is reverse. The strength of the magnetically induced current inside the rectangle $(R)$ in Fig. 1 is given by

$$
\begin{aligned}
I_{R} & =I_{l_{1}}\left[\mathbf{r}_{0} ; \mathbf{r}_{1}\right]+I_{l_{2}}\left[\mathbf{r}_{1} ; \mathbf{r}_{2}\right]+I_{l_{3}}\left[\mathbf{r}_{2} ; \mathbf{r}_{3}\right]+I_{l_{4}}\left[\mathbf{r}_{3} ; \mathbf{r}_{0}\right] \\
& =I_{l_{1}}\left[\mathbf{r}_{0} ; \mathbf{r}_{1}\right]+2 I_{l_{2}}\left[\mathbf{r}_{1} ; \mathbf{r}_{2}\right]
\end{aligned}
$$

yielding for $z_{0}>0$ the dependence of $I_{R}\left(z_{0}\right)$ as

$$
I_{R}\left(z_{0}\right)=\frac{1}{\mu_{0}}\left(\int_{0}^{z_{0}} \sigma_{z z}(0,0, z) \mathrm{d} z+\int_{0}^{\infty} \sigma_{x z}\left(x, 0, z_{0}\right) \mathrm{d} x\right)
$$

Eqn (8) gives the accumulated ring-current strength and approaches the strength of the global ring current in Table 1, when $z_{0} \rightarrow \infty$. The differential contribution or the ring-current profile is obtained by differentiating the accumulated ringcurrent strength as

$$
p\left(z_{0}\right)=\lim _{\varepsilon \rightarrow 0} \frac{I_{R}\left(z_{0}+\varepsilon\right)-I_{R}\left(z_{0}\right)}{\varepsilon} .
$$

The two identical ring-current profiles in the $z$ direction $(p(z))$ shown in Fig. 2 are obtained by integrating the current-density flux and by using eqn (8) and (9), respectively.

The ring-current profile in the $x$ direction $(p(x))$ can be analogously obtained by calculating the ring current passing through rectangular domains whose edge is stepwisely shifted along the $x$ axis. Alternatively, the strength of the current density inside the rectangular domain can also be obtained by integrating the appropriate elements of the magnetic

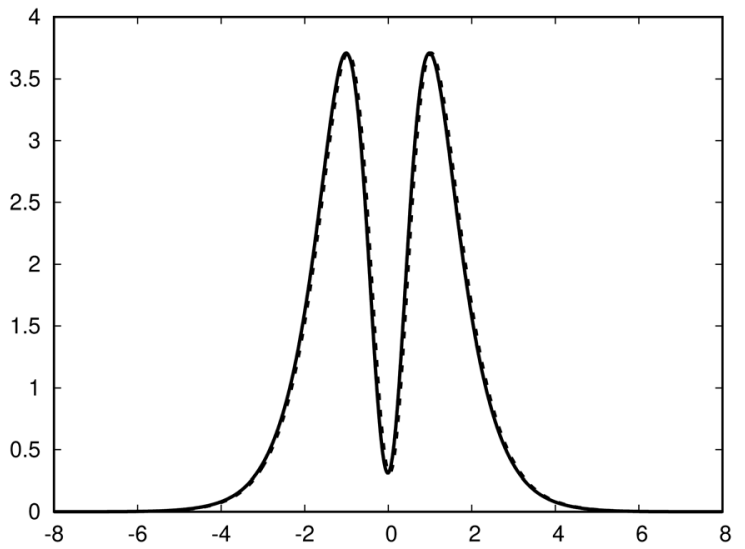

Fig. 2 The profile of the ring current (in $n A \mathrm{~T}^{-1}$ bohr $^{-1}$ ) of benzene $(p(z)$ ) along the $z$ axis. The solid line is obtained by integrating the current density. The dashed curve is obtained by integrating the magnetic shielding tensor. The dashed curve is slightly shifted to avoid that they are on top of each other.

shielding tensor along the edges of the rectangle. The integration domains along the sides of the rectangle are defined by the corners at $\mathbf{r}_{0}=(x, 0,-\infty), \mathbf{r}_{1}=(x, 0, \infty), \mathbf{r}_{2}=(\infty, 0,-\infty)$, and $\mathbf{r}_{3}=(\infty, 0, \infty)$. However, three of the contributions vanish since the induced magnetic field dies out at long distances from the molecule. The only remaining contribution is given by

$$
I_{R}(x)=\frac{1}{\mu_{0}} \int_{-\infty}^{\infty} \sigma_{z z}(x, 0, z) \mathrm{d} z
$$

yielding the ring-current strength as a function of $x$. The ringcurrent profiles in Fig. 3 are obtained by differentiating the integrated current-density flux $I_{R}(x)$ with respect to $x$. The profiles obtained using eqn (10) and (9) or by differentiating the integrated current density passing through the rectangular surface are identical. ${ }^{44}$ However, the one deduced from the shielding tensor has the correct sign in the whole range, whereas the one obtained from the current density changes sign on the other side of the ring-current vortex, because the returning ring current has the opposite sign due to charge conservation.

A relation between $\operatorname{NICS}_{z z}$ values at $z_{0}$ and the ring current can be obtained by differentiating eqn (8) with respect to $z$

$$
\operatorname{NICS}_{z z}\left(0,0, z_{0}\right)=-\mu_{0} p\left(z_{0}\right)+\left.2 \int_{0}^{\infty} \frac{\mathrm{d}}{\mathrm{d} z} \sigma_{x z}(x, 0, z)\right|_{z=z_{0}} \mathrm{~d} x
$$

where in the second term is of the derivative of the off-diagonal shielding tensor element $\sigma_{x z}$ with respect to $z$ that can be calculated using numerical differentiation.

\section{Conclusions and outlook}

We have shown that the strength of magnetically induced ring currents can be deduced from the $z z$ component of the magnetic shielding tensor calculated in discrete points along the $z$ axis. The approach has been demonstrated by calculating the ring-current strength of a few aromatic, antiaromatic and 


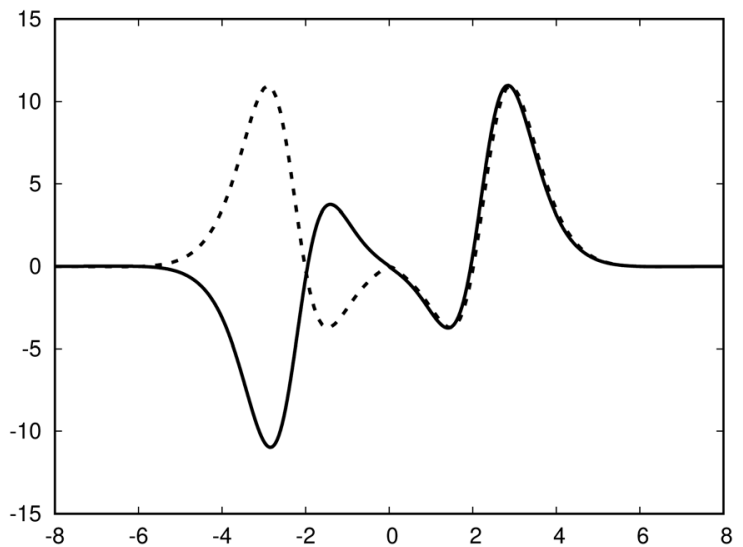

Fig. 3 The profile (in $n A \mathrm{~T}^{-1}$ bohr $^{-1}$ ) of the ring current $p(x)$ of benzene along the $x$ axis calculated from the magnetic shielding tensor using eqn (10) and (9) is shown with the dashed line. The ring-current profile deduced from the current density is shown with the solid line. The dashed curve is slightly shifted to avoid that they are on top of each other.

nonaromatic molecular rings. The theory is formally exact and the calculated ring-current strengths agree within numerical precision with values that were obtained by integrating the current-density flux circling around the molecular ring. The mean average error (MAE) is $0.0087 \mathrm{nA} \cdot \mathrm{T}^{-1}$ when using a spacing of $1 \mathrm{a}_{0}$.

The accumulated profile of the total ring current in the $z$ direction can be calculated in discrete points $\left(z_{0}\right)$ by integrating $\sigma_{x z}(x)$ in the interval $x \in[0, \infty]$ at $z_{0}$ and $\sigma_{z z}(z)$ in the interval $z \in\left[-z_{0}, z_{0}\right]$ along the $z$ axis. The accumulated radial ringcurrent profile along for example the $x$ axis can be calculated in discrete points $\left(x_{0}\right)$ along the $x$ axis by integrating $\sigma_{z z}(z)$ at $x=x_{0}$ in the interval $z \in[-\infty, \infty]$. The ring-current profiles are obtained by differentiating the accumulated ring-current profiles.

We also show that the popular $\operatorname{NICS}_{z z}(0)$ value has one contribution from the first derivative of the ring-current strength with respect to the $z$ direction and the second one is a non-local contribution consisting of an integral over the first derivative in the $z$ direction of the appropriate off-diagonal element of the magnetic shielding tensor calculated in the ring plane. The $\operatorname{NICS}_{z z}(1)$ value is analogously given by the first derivative of the ring-current strength with respect to the $z$ direction and the non-local contribution is calculated $1 \AA$ from the ring plane. Calculating magnetically induced current densities using NICS values on a three-dimensional grid is probably not feasible, ${ }^{14,45}$ whereas current-density strengths can be obtained from the magnetic shielding tensor calculated in discrete points. In our GIMIC method, we use the expression for magnetic shielding densities when we calculate magnetically induced current densities. ${ }^{19,21,39}$ NICS values and magnetic shielding tensors in discrete points can be obtained from magnetically induced current densities by integrating the BiotSavart expression. ${ }^{46-50}$

For molecular rings with lower symmetry, the exact ringcurrent strength can be obtained by integrating along the stagnation line i.e., the center of the global ring-current vortex instead of following the $z$ axis. Then, the induced magnetic field along the integration path is no longer restricted by the symmetry implying that also off-diagonal tensor elements such as $\sigma_{x z}$ and $\sigma_{y z}$ become non-zero and contribute to the ringcurrent strength.

The proposed methods to calculate ring-current strengths and ring-current profiles are easily employed in combination with electronic structure programs that provide nuclear magnetic resonance (NMR) shielding tensors. The present method should therefore contribute to settling the discussion about the choice of NICS points to assess molecular aromaticity. The ringcurrent strengths of three-dimensional molecules can be determined using our approach, when the external magnetic field is oriented in an appropriate direction for the system under consideration and the induced magnetic field is calculated along a closed path such that the studied ring current is enclosed by it. Depending on the direction of the external magnetic field and the path of the line integration, all elements of the magnetic shielding tensor may contribute to the ringcurrent strength. Calculating strengths of the current density inside carefully chosen integration paths may provide useful information about the current-density flux in complicated molecules.

The Ampère-Maxwell integration scheme combined with stagnation graph analysis can ultimately be used for calculating in an automatizable way the current strength of all vortices occurring in a molecule. Then, the line integral of the elements of the magnetic shielding tensor has to be evaluated along stagnation lines delimited by branching points. Some of us are currently working with their collaborators on the implementation of such a procedure.

\section{Author contributions}

R. B. conceived the idea to integrate the induced magnetic field along the stagnation lines of the current density. D. S. realized how to calculate ring-current profiles and ring-current strengths from magnetic shielding tensors. R. B. and D. S. developed the theory. M. D., R. N., and R. V. developed the computational methodology and performed the calculations. All authors discussed the results and contributed to the final manuscript.

\section{Conflicts of interest}

There are no conflicts to declare.

\section{Acknowledgements}

This work has been supported by the Academy of Finland through project numbers 314821 and 340583, the Finnish Cultural Foundation, the Swedish Cultural Foundation in Finland and by the DFG (German Research Foundation) in the Priority Program SPP1807 "Control of LD in molecular 
chemistry", BE4632/2-2. We acknowledge computational resources from the Finnish Grid and Cloud Infrastructure (persistent identifier urn:nbn:fi:research-infras-2016072533) and the CSC - IT Center for Science, Finland. R. R. V. thanks the Academy of Finland (340582) and is also thankful to the Russian Scientific Foundation (18-19-00268-P).

\section{Notes and references}

1 L. Pauling, J. Chem. Phys., 1936, 4, 673-677.

2 K. Lonsdale, Proc. R. Soc. (London) A, 1937, 159, 149-161.

3 F. London, J. Phys. Radium, 1937, 8, 397-409.

4 J. Pople, Mol. Phys., 1958, 1, 175-180.

5 R. McWeeny, Mol. Phys., 1958, 1, 311-321.

6 P. Lazzeretti, Prog. Nucl. Magn. Reson. Spectrosc., 2000, 36, $1-88$.

7 J. A. N. F. Gomes and R. B. Mallion, Chem. Rev., 2001, 101, 1349-1384.

8 C. Kumar, H. Fliegl and D. Sundholm, J. Phys. Chem. A, 2017, 121, 7282-7289.

9 P. von Ragué Schleyer, C. Maerker, A. Dransfeld, H. Jiao and N. J. R. van Eikema Hommes, J. Am. Chem. Soc., 1996, 118, 6317-6318.

10 J. Jusélius and D. Sundholm, Phys. Chem. Chem. Phys., 1999, 1, 3429-3435.

11 J. Jusélius and D. Sundholm, Phys. Chem. Chem. Phys., 2000, 2, 2145-2151.

12 Z. Chen, C. S. Wannere, C. Corminboeuf, R. Puchta and P. von Ragué Schleyer, Chem. Rev., 2005, 105, 3842-3888.

13 H. Fallah-Bagher-Shaidaei, C. S. Wannere, C. Corminboeuf, R. Puchta and P. von Ragué Schleyer, Org. Lett., 2006, 8, 863-866.

14 S. Klod and E. Kleinpeter, J. Chem. Soc., Perkin Trans. 2, 2001, 1893-1898.

15 G. Merino, T. Heine and G. Seifert, Chem. - Eur. J., 2004, 10, 4367-4371.

16 T. Heine, R. Islas and G. Merino, J. Comput. Chem., 2007, 28, 302-309.

17 R. Gershoni-Poranne and A. Stanger, Chem. - Eur. J., 2014, 20, 5673-5688.

18 M. Orozco-Ic, M. Dimitrova, J. Barroso, D. Sundholm and G. Merino, J. Phys. Chem. A, 2021, 125, 5753-5764.

19 J. Jusélius, D. Sundholm and J. Gauss, J. Chem. Phys., 2004, 121, 3952-3963.

20 H. Fliegl, S. Taubert, O. Lehtonen and D. Sundholm, Phys. Chem. Chem. Phys., 2011, 13, 20500-20518.

21 D. Sundholm, H. Fliegl and R. J. Berger, Wiley Interdiscip. Rev.: Comput. Mol. Sci., 2016, 639-678.

22 H. Fliegl, R. Valiev, F. Pichierri and D. Sundholm, in Chemical Modelling, Theoretical studies as a tool for understanding the aromatic character of porphyrinoid compounds, 2018, ch. 1, vol. 14, pp. 1-42.

23 M. Dimitrova and D. Sundholm, in Aromaticity: Modern Computational Methods and Applications, ed. I. F. López, Elsevier, 2021, ch. 5, pp. 155-194, DOI: 10.1016/C2019-004193-3, http://arxiv.org/abs/2105.04902.
24 D. Sundholm and H. Fliegl, in Handbook of Porphyrin Science, Vol 46, ed. K. M. Kadish, K. M. Smith and R. Guilard, World Scientific, 2022.

25 J. Jusélius and D. Sundholm, J. Org. Chem., 2000, 65, 5233-5237.

26 J. Jusélius and D. Sundholm, Phys. Chem. Chem. Phys., 2001, 3, 2433-2437.

27 A. C. Tsipis, Phys. Chem. Chem. Phys., 2009, 11, 8244-8261. 28 G. Monaco and R. Zanasi, J. Phys. Chem. A, 2014, 118, 1673-1683.

29 A. Stanger, J. Phys. Chem. A, 2019, 123, 3922-3927.

30 M. Jirásek, H. L. Anderson and M. D. Peeks, Acc. Chem. Res., 2021, 54, 3241-3251.

31 G. Monaco, R. Zanasi, S. Pelloni and P. Lazzeretti, J. Chem. Theory Comput., 2010, 6, 3343-3351.

32 A. D. Becke, Phys. Rev. A: At., Mol., Opt. Phys., 1988, 38, 3098-3100.

33 C. Lee, W. Yang and R. G. Parr, Phys. Rev. B: Condens. Matter Mater. Phys., 1988, 37, 785-789.

34 A. D. Becke, J. Chem. Phys., 1993, 98, 5648-5652.

35 F. Weigend and R. Ahlrichs, Phys. Chem. Chem. Phys., 2005, 7, 3297-3305.

36 S. Grimme, S. Ehrlich and L. Goerigk, J. Comput. Chem., 2011, 32, 1456-1465.

37 S. G. Balasubramani, G. P. Chen, S. Coriani, M. Diedenhofen, M. S. Frank, Y. J. Franzke, F. Furche, R. Grotjahn, M. E. Harding, C. Hättig, A. Hellweg, B. Helmich-Paris, C. Holzer, U. Huniar, M. Kaupp, A. Marefat Khah, S. Karbalaei Khani, T. Müller, F. Mack, B. D. Nguyen, S. M. Parker, E. Perlt, D. Rappoport, K. Reiter, S. Roy, M. Rückert, G. Schmitz, M. Sierka, E. Tapavicza, D. P. Tew, C. van Wüllen, V. K. Voora, F. Weigend, A. Wodynski and J. M. Yu, J. Chem. Phys., 2020, 152, 184107.

38 K. Reiter, F. Weigend, L. N. Wirz, M. Dimitrova and D. Sundholm, J. Phys. Chem. C, 2019, 123, 15354-15365.

39 GIMIC, version 2.0, a current density program, can be freely downloaded from https://github.com/qmcurrents/gimic, 2019.

40 Handbook of Mathematical Functions, ed. M. Abramowitz and I. A. Stegun, Dover, 1965.

41 G. Boole, A Treatise on the Calculus of Finite Differences, Dover, New York, 2003.

42 R. R. Valiev, I. Benkyi, Y. V. Konyshev, H. Fliegl and D. Sundholm, J. Phys. Chem. A, 2018, 122, 4756-4767.

43 S. Lehtola, M. Dimitrova, H. Fliegl and D. Sundholm, J. Chem. Theory Comput., 2021, 17, 1457-1468.

44 H. Fliegl, D. Sundholm, S. Taubert, J. Jusélius and W. Klopper, J. Phys. Chem. A, 2009, 113, 8668-8676.

45 S. Van Damme, G. Acke, R. W. A. Havenith and P. Bultinck, Phys. Chem. Chem. Phys., 2016, 18, 11746-11755.

46 R. M. Stevens, R. M. Pitzer and W. N. Lipscomb, J. Chem. Phys., 1963, 38, 550-560.

47 C. J. Jameson and A. D. Buckingham, J. Phys. Chem., 1979, 83, 3366-3371.

48 G. Acke, S. Van Damme, R. W. A. Havenith and P. Bultinck, Phys. Chem. Chem. Phys., 2019, 21, 3145-3153.

49 R. K. Jinger, H. Fliegl, R. Bast, M. Dimitrova, S. Lehtola and D. Sundholm, J. Phys. Chem. A, 2021, 125, 1778-1786.

50 H. Fliegl, M. Dimitrova, R. J. F. Berger and D. Sundholm, Chemistry, 2021, 3, 1005-1021. 